\title{
On Well-Posedness of 2D Dissipative Quasi-Geostrophic Equation in Critical Mixed Norm Lebesgue Spaces
}

\author{
Tuoc Phan ${ }^{1}$ and Yannick Sire ${ }^{2, *}$ \\ ${ }^{1}$ Department of Mathematics, University of Tennessee, 227 Ayres Hall, 1403 Circle \\ Drive, Knoxville, TN 37996-1320, USA \\ 2 Department of Mathematics, Johns Hopkins University, 404 Krieger Hall 3400 N. \\ Charles Street, Baltimore, MD 21218, USA
}

Received 12 November 2019; Accepted (in revised version) 28 April 2020

\begin{abstract}
We establish local and global well-posedness of the 2D dissipative quasigeostrophic equation in critical mixed norm Lebesgue spaces. The result demonstrates the persistence of the anisotropic behavior of the initial data under the evolution of the $2 \mathrm{D}$ dissipative quasi-geostrophic equation. The phenomenon is a priori nontrivial due to the nonlocal structure of the equation. Our approach is based on Kato's method using Picard's interation, which can be apdated to the multi-dimensional case and other nonlinear non-local equations. We develop time decay estimates for solutions of fractional heat equation in mixed norm Lebesgue spaces that could be useful for other problems.
\end{abstract}

Key Words: Local well-posedness, global well-posedness, dissipative quasi-geostrophic equation, fractional heat equation, mixed-norm Lebesgue spaces.

AMS Subject Classifications: 35A01, 35K55, 35K61

\section{Introduction and main result}

We study the Cauchy problem for the 2D dissipative quasi-geostrophic equation

$$
\begin{cases}u_{t}+(-\Delta)^{\alpha} u=\mathcal{R}(u) \cdot \nabla u & \text { in } \mathbb{R}^{2} \times(0, T), \\ u(\cdot, 0)=\theta_{0}(\cdot) & \text { in } \mathbb{R}^{2},\end{cases}
$$

where $\alpha \in(0,1), u: \mathbb{R}^{2} \times(0, T) \rightarrow \mathbb{R}$ is an unknown solution with some $T>0, \theta_{0}:$ $\mathbb{R}^{2} \rightarrow \mathbb{R}$ is a measurable function of initial data, and

$$
\mathcal{R}(u)=\left(-\mathcal{R}_{2}(u), \mathcal{R}_{1}(u)\right)
$$

*Corresponding author. Email addresses: phan@math.utk.edu (T. Phan), sire@math.jhu.edu (Y. Sire) 
in which $\mathcal{R}_{k}$ is the $k$ th Riesz transform which is defined by

$$
\mathcal{R}_{k}(u)=\partial_{x_{k}}(-\Delta)^{-\frac{1}{2}} u, \quad k=1,2 .
$$

Moreover, in (1.1), $(-\Delta)^{\alpha}$ denotes the fractional Laplace operator of order $\alpha$ whose precise definition will be recalled in Subsection 2.3.

The goal of this paper is to study the well-posedness of (1.1) in critical mixed-norm Lebesgue spaces. To make sense what we mean by this, let us recall the following scaling invariant property of (1.1). From a simple calculation, we see that for each solution $u$ of (1.1) and each $\lambda>0$, the rescaled function $u^{\lambda}$ defined by

$$
u^{\lambda}(x, t)=\lambda^{2 \alpha-1} u\left(\lambda x, \lambda^{2 \alpha} t\right), \quad(x, t) \in \mathbb{R}^{2} \times\left(0, T / \lambda^{2 \alpha}\right),
$$

is also a solution of (1.1) with the corresponding scaled initial data $\theta_{0}^{\lambda}$ defined as in (1.2).

Now, for each $p_{1}, p_{2} \in(1, \infty)$, the mixed norm $L_{p_{1}, p_{2}}\left(\mathbb{R}^{2}\right)$ of a measurable function $f: \mathbb{R}^{2} \rightarrow \mathbb{R}$ is defined in [1] by

$$
\|f\|_{L_{p_{1}, p_{2}}\left(\mathbb{R}^{2}\right)}=\left(\int_{\mathbb{R}}\left(\int_{\mathbb{R}}\left|f\left(x_{1}, x_{2}\right)\right|^{p_{1}} d x_{1}\right)^{\frac{p_{2}}{p_{1}}} d x_{2}\right)^{\frac{1}{p_{2}}} .
$$

Similar definitions can be formulated with either or both of $p_{1}=\infty, p_{2}=\infty$. Then, we observe that

$$
\left\|u^{\lambda}(\cdot, t)\right\|_{L_{p_{1}, p_{2}}\left(\mathbb{R}^{2}\right)}=\left\|u\left(\cdot, \lambda^{2 \alpha} t\right)\right\|_{L_{p_{1}, p_{2}}\left(\mathbb{R}^{2}\right)} \quad \text { for all } \lambda>0 \quad \text { and for all } t \in\left[0, T / \lambda^{2 \alpha}\right)
$$

if and only if

$$
\frac{1}{p_{1}}+\frac{1}{p_{2}}=2 \alpha-1
$$

Note that (1.3) is valid only when $\alpha \geq \frac{1}{2}$.

The study of (dissipative) active scalar equations has seen a great topic of research in the last decades, starting with the seminal works of Constantin, Majda and Tabak $[7,8]$. It is commonly known that (see also (1.3)), Eq. (1.1) is critical for $\alpha=\frac{1}{2}$, subcritical for $\alpha>\frac{1}{2}$ and supercritical otherwise. For the latter, the global well-posedness is largely open (see e.g., [24]). For the subcritical case, the problem has been investigated in [6] (see also e.g., [10,16]) and for the critical case in the seminal work [5] (see the predecessor paper [19] for smooth data and also [9]). The super-critical case has been addressed in [11, 12] where some regularity is assumed for the velocity. It is important to notice that even if in the present work we are considering a subcritical problem as far as the scaling is concerned, the fact that the initial data is chosen in a critical space does not allow to obtain easily a global well-posedness result for large data in our framework. Indeed, the local well-posedness in the critical Lebesgue space $L^{\frac{2}{2 \alpha-1}}$ obtained in [6] can be improved to a global one using the $L^{p}$-maximum principle in [13]. However in our case, we do not 
know if such a maximum principle in mixed Lebesgue norms is available, leaving open the global well-posedness for large data. We plan to address this issue in the next future.

There is by now a huge literature dealing with this type of equations with or without dissipation and we refer the reader to the excellent survey paper [18].

In this paper, we focus on well-posedness of (1.1) in critical mixed norm spaces $L_{p_{1}, p_{2}}\left(\mathbb{R}^{2}\right)$ in which $p_{1}, p_{2}$ satisfy (1.3). In particular, the results of the paper demonstrate the persistence of the anisotropic behavior of the initial data under the evolution of the 2D dissipative quasi-geostrophic equation. Due to the nonlocal structure of the equation, the phenomenon is a priori nontrivial and interesting. To state our result precisely, we introduce some notations. Given $p=\left(p_{1}, p_{2}\right)$ and $q=\left(q_{1}, q_{2}\right)$ such that $p_{k} \in(1, \infty)$ and $q_{k} \in\left[p_{k}, \infty\right)$ for $k=1,2$, assume that (1.3) holds and

$$
\frac{1}{q_{1}}+\frac{1}{q_{2}}=\delta \in(0,2 \alpha-1)
$$

Then, given $T \in(0, \infty]$, we denote $X_{p, q, T}$ the space consisting of all measurable functions $f: \mathbb{R}^{2} \times[0, T) \rightarrow \mathbb{R}$ such that for

$$
g(x, t)=t^{\frac{2 \alpha-1-\delta}{2 \alpha}} f(x, t) \text { and } \quad \tilde{g}(x, t)=t^{\frac{1}{2 \alpha}} \nabla f(x, t) \quad \text { with }(x, t) \in \mathbb{R}^{2} \times(0, T),
$$

then

$$
g \in \mathrm{C}\left([0, T), L_{q_{1}, q_{2}}\left(\mathbb{R}^{2}\right)\right), \quad \tilde{g} \in \mathrm{C}\left([0, T), L_{p_{1}, p_{2}}\left(\mathbb{R}^{2}\right)^{2}\right),
$$

and moreover $g(x, 0)=0, \tilde{g}(x, 0)=0$ and the norm

$$
\|f\|_{X_{p, q, T}}=\sup _{t \in(0, T)}\left[\|g(\cdot, t)\|_{L_{q_{1}, q_{2}}\left(\mathbb{R}^{2}\right)}+\|\tilde{g}\|_{L_{p_{1}, p_{2}}\left(\mathbb{R}^{2}\right)}\right]<\infty .
$$

We also denote $y_{p, T}$ the space consisting of all functions $f \in C\left([0, T), L_{p_{1}, p_{2}}\left(\mathbb{R}^{2}\right)\right)$ such that $t^{\frac{1}{2 \alpha}} \nabla f \in C\left([0, T), L_{p_{1}, p_{2}}\left(\mathbb{R}^{2}\right)^{2}\right)$ and

$$
\|f\|_{y_{p, T}}=\sup _{t \in(0, T)}\left[\|f(t)\|_{L_{p_{1}, p_{2}}\left(\mathbb{R}^{2}\right)}+t^{\frac{1}{2 \alpha}}\|\nabla f(t)\|_{L_{p_{1}, p_{2}}\left(\mathbb{R}^{2}\right)}\right]<\infty .
$$

The following result exhibits local and global existence together with uniqueness in mixed norm Lebesgue spaces $L_{p_{1}, p_{2}}\left(\mathbb{R}^{2}\right)$.

Theorem 1.1. Let $\alpha \in\left(\frac{1}{2}, 1\right], p=\left(p_{1}, p_{2}\right)$ and $q=\left(q_{1}, q_{2}\right)$ with $p_{k} \in(1, \infty), q_{k} \in\left[p_{k}, \infty\right)$ for $k=1,2$. Assume also that the conditions (1.3) and (1.4) hold and

$$
\frac{p_{k}}{q_{k}}+1 \leq p_{k}, \quad k=1,2 .
$$

Then, there exist a sufficiently small constant $\lambda_{0}>0$ and a large number $N_{0}>0$ depending only on $\alpha, p$, and $q$ such that the following assertions hold. 
(i) For every $\theta_{0} \in L_{p_{1}, p_{2}}\left(\mathbb{R}^{2}\right)$, if $\left\|\theta_{0}\right\|_{L_{p_{1}, p_{2}}\left(\mathbb{R}^{2}\right)} \leq \lambda_{0}$, then the Cauchy problem (1.1) has unique global in time solution $u \in X_{p, q, \infty} \cap y_{p, \infty}$ with

$$
\|u\|_{x_{p, q, \infty}} \leq N_{0}\left\|\theta_{0}\right\|_{L_{p_{1}, p_{2}}\left(\mathbb{R}^{2}\right)} \text { and }\|u\|_{y_{p, \infty}} \leq N_{0}\left\|\theta_{0}\right\|_{L_{p_{1}, p_{2}}\left(\mathbb{R}^{2}\right)}
$$

(ii) For every $\theta_{0} \in L_{p_{1}, p_{2}}\left(\mathbb{R}^{2}\right)$, there exists $T_{0}>0$ sufficiently small depending on $\alpha, p$, $q$ and $\theta_{0}$ such that the Cauchy problem (1.1) has unique local in time solution $u \in x_{p, q, T_{0}} \cap y_{p, T_{0}}$ with

$$
\|u\|_{x_{p, q, T_{0}}} \leq N_{0}\left\|\theta_{0}\right\|_{L_{p_{1}, p_{2}}\left(\mathbb{R}^{2}\right)} \text { and } \quad\|u\|_{y_{p, T_{0}}} \leq N_{0}\left\|\theta_{0}\right\|_{L_{p_{1}, p_{2}}\left(\mathbb{R}^{2}\right)}
$$

The previous theorem is proved via the Kato's method which is based on the Picard's iteration. A straightforward inspection of the proof of Theorem 1.1 shows that similar results also hold if in (1.1) we replace $\mathbb{R}^{2}$ by $\mathbb{R}^{n}$ for any $n \geq 2$, and $\mathcal{R}$ is any operator so that each of its components is bounded in weighted Lebesgue space $L_{p}\left(\mathbb{R}^{n}, \omega\right) \rightarrow L_{p}\left(\mathbb{R}^{n}, \omega\right)$ for any Muckenthoupt $A_{p}$ weight $\omega$, like for instance a Calderon-Zygmund operator (see Section 2.1 for its definition). It is also possible to extend the results and ideas in this work to more general equations and systems of equations in more general setting of mixed-norm functional spaces such as mixed norm Sobolev spaces and mixed norm Besov spaces.

\section{Preliminary estimates in mixed norm Lebesgue spaces}

For $p_{1}, \cdots, p_{n} \in[1, \infty)$, and for a given measurable function $f: \mathbb{R}^{n} \rightarrow \mathbb{R}$, we say that $f$ belongs to the mixed-norm Lebesgue space $L_{p_{1}, \cdots, p_{n}}\left(\mathbb{R}^{n}\right)$ if its norm

$$
\begin{aligned}
& \|f\|_{L_{p_{1}, \cdots, p_{n}}\left(\mathbb{R}^{n}\right)} \\
= & \left(\left(\left(\cdots\left(\int_{\mathbb{R}}\left|f\left(x_{1}, \cdots x_{n}\right)\right|^{p_{1}} d x_{1}\right)^{\frac{p_{2}}{p_{1}}} d x_{2} \cdots\right)^{\frac{p_{n-1}}{p_{n-2}}} d x_{n-1}\right)^{\frac{p_{n}}{p_{n-1}}} d x_{n}\right)^{\frac{1}{p_{n}}}<\infty .
\end{aligned}
$$

Similar definitions can be also formulated if some of the indices in $\left\{p_{1}, \cdots, p_{n}\right\}$ are equal to $\infty$. Note that it follows directly from the definition that if $p=p_{1}=\cdots=p_{n}$, then $L_{p_{1}, \cdots, p_{n}}\left(\mathbb{R}^{n}\right)$ is the same as the usual Lebesgue space $L_{p}\left(\mathbb{R}^{n}\right)$. For more details on mixed norm Lebesgue space $L_{p_{1}, \cdots, p_{n}}\left(\mathbb{R}^{n}\right)$, one can see [1].

\subsection{Riesz transform in mixed norm spaces}

This subsection proves that the Riesz transforms are bounded in the mixed-norm Lebesgue spaces. The main result of the section is stated in the following theorem. 
Theorem 2.1. For any $j=1, \cdots, n$ and any $p_{1}, \cdots, p_{n} \in(1, \infty)$, there exists a positive constant $N=N\left(p_{1}, \cdots, p_{n}, n\right)$ such that

$$
\left\|\mathcal{R}_{j}(f)\right\|_{L_{p_{1}, \cdots, p_{n}}\left(\mathbb{R}^{n}\right)} \leq N\|f\|_{L_{p_{1}, \cdots, p_{n}}\left(\mathbb{R}^{n}\right)}
$$

for every $f \in L_{p_{1}, \cdots, p_{n}}\left(\mathbb{R}^{n}\right)$, where $\mathcal{R}_{j}$ is the $j^{\text {th }}$-Riesz transform defined by $\mathcal{R}_{j}(f)=$ $\partial_{x_{j}}(-\Delta)^{-\frac{1}{2}} f$.

To prove Theorem 2.1, it requires several definitions and results from analysis. We first recall the definition of Muckenhoupt $A_{q}\left(\mathbb{R}^{n}\right)$-class of weights. For each $q \in(1, \infty)$, a non-negative, locally integrable function $\omega: \mathbb{R}^{n} \rightarrow \mathbb{R}$ is said to be in the Muckenhoupt $A_{q}\left(\mathbb{R}^{n}\right)$-class of weights if

$$
[\omega]_{A_{q}}=: \sup _{R>0, x_{0} \in \mathbb{R}^{n}}\left(\frac{1}{\left|B_{R}\left(x_{0}\right)\right|} \int_{B_{R}\left(x_{0}\right)} \omega(x) d x\right)\left(\frac{1}{\left|B_{R}\left(x_{0}\right)\right|} \int_{B_{R}\left(x_{0}\right)} \omega(x)^{-\frac{1}{q-1}} d x\right)^{q-1}<\infty,
$$

where $B_{R}\left(x_{0}\right)$ denotes the ball in $\mathbb{R}^{n}$ of radius $R$ centered at $x_{0} \in \mathbb{R}^{n}$. In the following, for each given $p \in[1, \infty)$ and each given weight $\omega: \mathbb{R}^{n} \rightarrow \mathbb{R}$, a measurable function $f: \mathbb{R}^{n} \rightarrow \mathbb{R}$ is said to be in the weighted Lebesgue space $L_{p}\left(\mathbb{R}^{n}, \omega\right)$ if its norm

$$
\|f\|_{L_{p}\left(\mathbb{R}^{n}, \omega\right)}=\left(\int_{\mathbb{R}^{n}}|f(x)|^{p} \omega(x) d x\right)^{\frac{1}{p}}<\infty .
$$

We recall the following amazing result from [21, Theorem 6.2], which is a beautiful application of the Rubio De Francia extrapolation theory (see [14] and [15, Corollary 2.7] for instance).

Theorem 2.2. Let $p_{k} \in(1, \infty)$ for all $k=1, \cdots, n$. Then, there exists a constant $K_{0}=$ $K_{0}\left(n, p_{1}, \cdots, p_{n}\right)$ such that the following holds true. For a pair of given measurable functions $f, g: \mathbb{R}^{n} \rightarrow \mathbb{R}$ such that if

$$
\|f\|_{L_{p_{1}}\left(\mathbb{R}^{n}, \omega\right)} \leq\|g\|_{L_{p_{1}}\left(\mathbb{R}^{n}, \omega\right)}
$$

for every $\omega \in A_{p_{1}}$ with $[\omega]_{A_{p_{1}}} \leq K_{0}$, then we have

$$
\|f\|_{L_{p_{1}, \cdots, p_{n}}\left(\mathbb{R}^{n}\right)} \leq 4^{n}\|g\|_{L_{p_{1}, \cdots, p_{n}}\left(\mathbb{R}^{n}\right)} .
$$

Now, we are ready to provide the proof of Theorem 2.1.

Proof of Theorem 2.1. We plan to apply Theorem 2.2. For given $p_{1}, \cdots, p_{n} \in(1, \infty)$, let $K_{0}$ be as in Theorem 2.2. By using the truncation and a multiplication with suitable cutoff functions, we can approximate $f \in L_{p_{1}, \cdots, p_{n}}\left(\mathbb{R}^{n}\right)$ by a sequence of bounded compactly supported functions (see [1]). Therefore, we may assume that $f$ is bounded and compactly supported in $\mathbb{R}^{n}$. Without loss of generality, we can also assume that 
$p_{1}=\min \left\{p_{1}, \cdots, p_{n}\right\}$. Under these assumptions, we see that $f \in L_{p_{1}}\left(\mathbb{R}^{n}, \omega\right)$ for every weight $\omega \in A_{p_{1}}\left(\mathbb{R}^{n}\right)$. Then, since $p_{1} \in(1, \infty)$, by the classical Calderón-Zygmund theory (see [14,17] for instance), there exists a constant $N=N\left(p_{1}, n, K_{0}\right)$ such that

$$
\left\|\mathcal{R}_{j}(f)\right\|_{L_{p_{1}}\left(\mathbb{R}^{n}, \omega\right)} \leq N\|f\|_{L_{p_{1}}\left(\mathbb{R}^{n}, \omega\right)}
$$

for every $\omega \in A_{p_{1}}$ with $[\omega]_{A_{p_{1}}} \leq K_{0}$. From (2.1) and Theorem 2.2, we infer that

$$
\left\|\mathcal{R}_{j}(f)\right\|_{L_{p_{1}, \cdots, p_{n}}\left(\mathbb{R}^{n}\right)} \leq 4^{n} N\|f\|_{L_{p_{1}, \cdots, p_{n}}\left(\mathbb{R}^{n}\right)} .
$$

This is the desired estimate and the proof is therefore completed.

\subsection{Young's inequality in mixed norm Lebesgue spaces}

The following Young's inequality in mixed norm Lebesgue spaces is needed in this paper.

Proposition 2.1 (Young's inequality in mixed norm). Let $p_{k}, r_{k}$ and $q_{k}$ be given numbers in $[1, \infty]$ that satisfy

$$
\frac{1}{p_{k}}+1=\frac{1}{q_{k}}+\frac{1}{r_{k}}, \quad k=1, \cdots, n
$$

Then

$$
\|f * g\|_{L_{p_{1}, \cdots, p_{n}}\left(\mathbb{R}^{n}\right)} \leq\|f\|_{L_{q_{1}, \cdots, q_{n}}\left(\mathbb{R}^{n}\right)}\|g\|_{L_{r_{1}, \cdots, r_{n}}\left(\mathbb{R}^{n}\right)}
$$

for every $f \in L_{q_{1}, \cdots, q_{n}}\left(\mathbb{R}^{n}\right)$ and $g \in L_{r_{1}, \cdots, r_{n}}\left(\mathbb{R}^{n}\right)$.

Proof. The proof can be done using induction on the dimension $n$. For details, see [23, Theorem 1.2].

\subsection{Fractional heat equations in mixed norm Lebesgue spaces}

We consider the Cauchy problem of the fractional heat equation

$$
\begin{cases}u_{t}+(-\Delta)^{s} u=0 & \text { in } \mathbb{R}^{n} \times(0, \infty), \\ u(x, 0)=u_{0}(x) & \text { for } x \in \mathbb{R}^{n}\end{cases}
$$

where $s \in(0,1]$ is a given number and $u_{0}: \mathbb{R}^{n} \rightarrow \mathbb{R}$ is a given measurable function. The fractional Laplace operator $(-\Delta)^{s}$ can be defined through its Fourier transform or by the presentation (see [25] for instance)

$$
(-\Delta)^{s} f(x)=N(n, s) \int_{\mathbb{R}^{n}} \frac{f(x)-f(y)}{|x-y|^{2 n+2 s}} d y .
$$

Under some sufficient conditions on the given initial data $u_{0}$, the function

$$
u(x, t)=e^{-(-\Delta)^{s} t} u_{0}(x)=\int_{\mathbb{R}^{n}} P_{s}(x-y, t) u_{0}(y) d y
$$


is a solution of (2.3). Here, in (2.4) the function $P_{S}(x, t)$ is the fractional heat kernel. When $s=1$, the fractional heat equation becomes the usual heat equation and

$$
P_{1}(x, t)=\frac{1}{(4 \pi t)^{\frac{n}{2}}} e^{-\frac{|x|^{2}}{4 t}}, \quad(x, t) \in \mathbb{R}^{n} \times(0, \infty) .
$$

For general $s \in(0,1)$ the formula of $P_{s}(x, t)$ is not explicit, except for the case $s=\frac{1}{2}$ for which

$$
P_{\frac{1}{2}}(x, t)=\frac{N(n) t}{\left(t^{2}+|x|^{2}\right)^{\frac{n+1}{2}}}, \quad(x, t) \in \mathbb{R}^{n} \times(0, \infty),
$$

where $N(n)=\Gamma(n+(1 / 2)) / \pi^{n+(1 / 2)}$ is a normalization constant. However, for every $s \in(0,1)$, it is known from $[3,20]$ that $P_{s} \in C^{\infty}\left(\mathbb{R}^{n} \times(0, \infty)\right)$. Moreover, it also follows from [2] that

$$
P_{s}(x, t)=t^{-\frac{n}{2 s}} F_{S}\left(|x| t^{-\frac{1}{2 s}}\right),
$$

where $F_{s} \in C^{\infty}(\mathbb{R})$ satisfying the following asymptotic property

$$
\lim _{|\xi| \rightarrow \infty}|\xi|^{n+2 s} F_{s}(\xi)=N(n, s) .
$$

In particular, for $s \in(0,1)$, we have

$$
\frac{N_{1}(n, s) t}{\left(t^{\frac{1}{s}}+|x|^{2}\right)^{\frac{n+2 s}{2}}} \leq P_{s}(x, t) \leq \frac{N_{2}(n, s) t}{\left(t^{\frac{1}{s}}+|x|^{2}\right)^{\frac{n+2 s}{2}}}, \quad(x, t) \in \mathbb{R}^{n} \times(0, \infty),
$$

for some universal positive constants $N_{1}, N_{2}$.

The main result of this section is the following theorem on time decay estimates for the fractional heat equation in mixed norm Lebesgue spaces.

Theorem 2.3 (Time decaying of solutions for fractional heat equation in mixed-norm). Let $s \in(0,1]$ and $1 \leq q_{k} \leq p_{k} \leq \infty$. There exists a positive constant $N$ depending only on $p_{1}, \cdots, p_{n}, q_{1}, \cdots, q_{n}, s$ and $n$ such that for the solution $u(x, t)=e^{-(-\Delta)^{s} t} u_{0}(x)$ defined in (2.4) of the Cauchy problem (2.3) with $u_{0} \in L_{q_{1}, \cdots, q_{n}}\left(\mathbb{R}^{n}\right)$, it holds that

$$
\|u(\cdot, t)\|_{L_{p_{1}, \cdots, p_{n}}\left(\mathbb{R}^{n}\right)} \leq N t^{-\frac{1}{2 s} \sum_{k=1}^{n}\left(\frac{1}{q_{k}}-\frac{1}{p_{k}}\right)}\left\|u_{0}\right\|_{L_{q_{1}, \cdots, q_{n}}\left(\mathbb{R}^{n}\right)} \text { for } t>0 .
$$

Moreover, for every $l=1, \cdots$ and for $t>0$

$$
\left\|D_{x}^{l} u(\cdot, t)\right\|_{L_{p_{1}, \cdots, p_{n}}\left(\mathbb{R}^{n}\right)} \leq N t^{-\frac{l}{2 s}-\frac{1}{2 s} \sum_{k=1}^{n}\left(\frac{1}{q_{k}}-\frac{1}{p_{k}}\right)}\left\|u_{0}\right\|_{L_{q_{1}, \cdots, q_{n}}\left(\mathbb{R}^{n}\right)},
$$

where $D_{x}^{l}$ denotes the $l^{\text {th }}$-derivative in $x$-variable. 
Proof. When $s=1$, Theorem 2.3 follows from [23, Theorem 2.9]. Therefore, we only consider the case $s \in(0,1)$. We begin with the proof of (2.6). For each $k=1, \cdots, n$, by the assumption that $q_{k} \leq p_{k}$, we can find $r_{k} \in[1, \infty]$ such that

$$
\frac{1}{p_{k}}+1=\frac{1}{r_{k}}+\frac{1}{q_{k}}
$$

Then, because $u(x, t)=\left(P_{s}(\cdot, t) * u_{0}\right)(x)$, we can use the mixed-norm Young's inequality in Theorem 2.1 to see that

$$
\|u(\cdot, t)\|_{L_{p_{1}, \cdots, p_{n}}\left(\mathbb{R}^{n}\right)} \leq\left\|P_{S}(\cdot, t)\right\|_{L_{r_{1}, \cdots, r_{n}}\left(\mathbb{R}^{n}\right)}\left\|u_{0}\right\|_{L_{q_{1}, \cdots, q_{n}}\left(\mathbb{R}^{n}\right)} .
$$

We now estimate $\left\|P_{\mathcal{S}}(\cdot, t)\right\|_{L_{r_{1}, \cdots, r_{n}}\left(\mathbb{R}^{n}\right)}$. By $(2.5)$, we see that

$$
\left\|P_{S}(\cdot, t)\right\|_{L_{r_{1}, \cdots, r_{n}}\left(\mathbb{R}^{n}\right)} \leq N(n, s) t^{-\frac{n}{2 s}}\left\|G_{t}(\cdot)\right\|_{L_{r_{1}, \cdots, r_{n}}\left(\mathbb{R}^{n}\right)},
$$

where

$$
G_{t}(x)=\frac{1}{\left(1+\left|\frac{x}{t \frac{1}{2 s}}\right|^{2}\right)^{\frac{n+2 s}{2}}} .
$$

Then, by the change of variable $y=\frac{x}{t^{\frac{1}{2 s}}}$, we see that

$$
\begin{aligned}
\left\|G_{t}(\cdot)\right\|_{L_{r_{1}, \cdots, r_{n}}\left(\mathbb{R}^{n}\right)} & =t^{\frac{1}{2 s} \sum_{k=1}^{n} \frac{1}{r_{k}}}\left\|G_{1}(\cdot)\right\|_{L_{r_{1}, \cdots, r_{n}}\left(\mathbb{R}^{n}\right)} \\
& =N\left(n, r_{1}, \cdots, r_{n}\right) t^{\frac{1}{2 s}\left[n-\sum_{k=1}^{n}\left(\frac{1}{q_{k}}-\frac{1}{p_{k}}\right)\right]} .
\end{aligned}
$$

This last estimate and (2.9) imply (2.6).

Next, we prove (2.7). We only demonstrate the proof of (2.7) with $l=1$ as the general case can be done in a similar way. We observe that for each $i=1, \cdots, n$,

$$
D_{x_{i}} u(x, t)=\left(\left[D_{x_{i}} P_{s}(\cdot, t)\right] * u_{0}\right)(x), \quad(x, t) \in \mathbb{R}^{n} \times(0, \infty) .
$$

Then, by the mixed norm Young's inequality in Theorem 2.1, we have

$$
\left\|D_{x_{i}} u(\cdot, t)\right\|_{L_{p_{1}, \cdots, p_{n}}\left(\mathbb{R}^{n}\right)} \leq\left\|D_{x_{i}} P_{S}(\cdot, t)\right\|_{L_{r_{1}, \cdots, r_{n}}\left(\mathbb{R}^{n}\right)}\left\|u_{0}(\cdot)\right\|_{L_{q_{1}, \cdots, q_{n}}\left(\mathbb{R}^{n}\right)} .
$$

It remains to estimate the mixed norm $\left\|D_{x_{i}} P_{S}(\cdot, t)\right\|_{L_{r_{1}, \cdots, r_{n}}\left(\mathbb{R}^{n}\right)}$. Observe that (see [4, Eq. (1.5)] for instance)

$$
\left|D_{x_{i}} P_{s}(x, t)\right| \leq \frac{N(n, s) t x_{i}}{\left(t^{\frac{1}{s}}+|x|^{2}\right)^{\frac{n+2 s}{2}}+1}=N(n, s) t^{-\frac{n}{2 s}-\frac{1}{2 s}} \frac{\frac{x_{i}}{t^{\frac{1}{2 s}}}}{\left(1+\left|\frac{x}{t^{\frac{1}{2 s}}}\right|^{2}\right)^{\frac{n+2 s}{2}+1}}
$$


Then, by using the change of variable $x \mapsto \frac{x}{t^{\frac{1}{25}}}$, we see that

$$
\begin{aligned}
\left\|D_{x_{i}} P_{S}(\cdot, t)\right\|_{L_{r_{1}, \cdots, r_{n}}\left(\mathbb{R}^{n}\right)} & \leq N\left(n, s, r_{1}, \cdots, r_{n}\right) t^{-\frac{n}{2 s}-\frac{1}{2 s}} t^{\frac{1}{2 s} \sum_{k=1}^{n} \frac{1}{r_{k}}} \\
& =N\left(n, s, r_{1}, \cdots, r_{n}\right) t^{-\frac{1}{2 s}-\frac{1}{2 s} \sum_{k=1}^{n}\left(\frac{1}{q_{k}}-\frac{1}{p_{k}}\right)} .
\end{aligned}
$$

From this, (2.7) with $l=1$ follows. The proof is completed.

Next, we introduce and prove the following simple lemma on the continuity property of the solutions of the fractional heat equation (2.3) in mixed norm spaces.

Lemma 2.1. Let $s \in(0,1]$ and $p_{k} \in[1, \infty)$ for $k=1, \cdots, n$. Assume that $u_{0} \in L_{p_{1}, \cdots, p_{n}}\left(\mathbb{R}^{n}\right)$. Let $u(x, t)=e^{-(-\Delta)^{s} t} u_{0}$ be the solution of the fractional heat equation (2.3) defined in (2.4). Then, the following assertions hold

(i) $u \in C\left([0, \infty), L_{p_{1}, \cdots, p_{n}}\left(\mathbb{R}^{n}\right)\right)$ and

$$
\lim _{t \rightarrow 0^{+}}\left\|u(\cdot, t)-u_{0}\right\|_{L_{p_{1}, \cdots, p_{n}}\left(\mathbb{R}^{n}\right)}=0 .
$$

(ii) For every $q_{k} \in\left[p_{k}, \infty\right)$ with $k=1, \cdots, n$, let

$$
\sigma=\sum_{k=1}^{n}\left(\frac{1}{p_{k}}-\frac{1}{q_{k}}\right)
$$

we have

$$
\begin{aligned}
& \lim _{t \rightarrow 0^{+}} t^{\frac{\sigma}{2 \alpha}}\|u(\cdot, t)\|_{L_{q_{1}, \cdots, q_{n}}\left(\mathbb{R}^{n}\right)}=0, \\
& \lim _{t \rightarrow 0^{+}} t^{\frac{1+\sigma}{2 \alpha}}\|\nabla u(\cdot, t)\|_{L_{q_{1}, \cdots, q_{n}}\left(\mathbb{R}^{n}\right)}=0 .
\end{aligned}
$$

Proof. We start with proving the assertion (i). We only prove (2.11) as the proof of the continuity of $u$ at $t_{0}>0$ can be done similarly. Let $\epsilon>0$, from [1], by using the truncation and a multiplication by a suitable cut-off function, we can find a bounded compactly support function $\tilde{u}_{0}$ such that

$$
\left\|u_{0}-\tilde{u}_{0}\right\|_{L_{p_{1}, \cdots, p_{n}}\left(\mathbb{R}^{n}\right)} \leq \frac{\epsilon}{4 N}
$$

where $N=N\left(n, p_{1}, \cdots, p_{n}\right)>1$ is the number defined in Theorem 2.3. Then, by Theorem 2.3 , we have

$$
\left\|e^{-(-\Delta)^{s} t}\left(u_{0}-\tilde{u}_{0}\right)\right\|_{L_{p_{1}, \cdots, p_{n}}\left(\mathbb{R}^{n}\right)} \leq N_{0}\left\|u_{0}-\tilde{u}_{0}\right\|_{L_{p_{1}, \cdots, p_{n}}\left(\mathbb{R}^{n}\right)} \leq \frac{\epsilon}{4} .
$$


From the previous two estimates, we see that

$$
\left\|e^{-(-\Delta)^{s} t}\left(u_{0}-\tilde{u}_{0}\right)-\left(u_{0}-\tilde{u}_{0}\right)\right\|_{L_{p_{1}, \cdots, p_{n}}\left(\mathbb{R}^{n}\right)} \leq \frac{\epsilon}{4}+\frac{\epsilon}{4 N} \leq \frac{\epsilon}{2} .
$$

Our next goal is to show that

$$
\lim _{t \rightarrow 0^{+}}\left\|e^{-(-\Delta)^{s} t} \tilde{u}_{0}-\tilde{u}_{0}\right\|_{L_{p_{1}, \cdots, p_{n}}\left(\mathbb{R}^{n}\right)}=0 .
$$

Take $p>\max \left\{p_{1}, \cdots, p_{n}\right\}$ and choose the numbers $q_{k} \in\left(p_{k}, \infty\right)$ such that

$$
\frac{1}{q_{k}}=\frac{1}{p_{k}}-\frac{1}{p^{\prime}}, k=1, \cdots, n .
$$

Then, by applying the Hölder's inequality repeatedly for each integration with respect to each variable $x_{k}$, we see that

$$
\begin{aligned}
& \left\|e^{-(-\Delta)^{s} t} \tilde{u}_{0}-\tilde{u}_{0}\right\|_{L_{p_{1}, \cdots, p_{n}}\left(\mathbb{R}^{n}\right)} \\
\leq & \left\|e^{-(-\Delta)^{s} t} \tilde{u}_{0}-\tilde{u}_{0}\right\|_{L_{p}\left(\mathbb{R}^{n}\right)}\left\|e^{-(-\Delta)^{s} t} \tilde{u}_{0}-\tilde{u}_{0}\right\|_{L_{q_{1}, \cdots, q_{n}}\left(\mathbb{R}^{n}\right)} \\
\leq & \left\|e^{-(-\Delta)^{s} t} \tilde{u}_{0}-\tilde{u}_{0}\right\|_{L_{p}\left(\mathbb{R}^{n}\right)}\left[\left\|e^{-(-\Delta)^{s} t} \tilde{u}_{0}\right\|_{L_{q_{1}, \cdots, q_{n}}\left(\mathbb{R}^{n}\right)}+\left\|\tilde{u}_{0}\right\|_{L_{q_{1}, \cdots, q_{n}}\left(\mathbb{R}^{n}\right)}\right] \\
\leq & N\left\|e^{-(-\Delta)^{s} t} \tilde{u}_{0}-\tilde{u}_{0}\right\|_{L_{p}\left(\mathbb{R}^{n}\right)}\left\|\tilde{u}_{0}\right\|_{L_{q_{1}, \cdots, q_{n}}\left(\mathbb{R}^{n}\right)} .
\end{aligned}
$$

Observe that as $\tilde{u}_{0}$ is bounded and compactly supported, $\left\|\tilde{u}_{0}\right\|_{L_{q_{1}, \cdots, q_{n}}\left(\mathbb{R}^{n}\right)}<\infty$. Therefore,

$$
\left\|e^{-(-\Delta)^{s} t} \tilde{u}_{0}-\tilde{u}_{0}\right\|_{L_{p_{1}, \cdots, p_{n}}\left(\mathbb{R}^{n}\right)} \leq \tilde{N}\left\|e^{-(-\Delta)^{s} t} \tilde{u}_{0}-\tilde{u}_{0}\right\|_{L_{p}\left(\mathbb{R}^{n}\right)} \rightarrow 0 \text { as } t \rightarrow 0^{+},
$$

where in the last assertion, we used the continuity of the fractional heat flow in the unmixed space $L_{p}\left(\mathbb{R}^{n}\right)$ and the fact that $\tilde{u}_{0} \in L_{p}\left(\mathbb{R}^{n}\right)$. From this and (2.13), we conclude that there is $\delta=\delta(\epsilon)>0$ such that

$$
\left\|e^{-(-\Delta)^{s} t} u_{0}-u_{0}\right\|_{L_{p_{1}, \cdots, p_{n}}\left(\mathbb{R}^{n}\right)} \leq \epsilon, \quad \forall t \in\left(0, \delta_{0}\right) .
$$

This proves (2.11) as desired.

We now prove (ii). We only provide the proof of the first assertion in (2.12) as the second one can be done similarly. We may assume that $u_{0}$ is bounded and compactly supported if needed. Let $\epsilon>0$. Then, by using approximation, we can find $g \in L_{p_{1}, \cdots, p_{n}}\left(\mathbb{R}^{n}\right)^{n} \cap L_{q_{1}, \cdots, q_{n}}\left(\mathbb{R}^{n}\right)^{n}$ such that

$$
\left\|u_{0}-g\right\|_{L_{p_{1}, \cdots, p_{n}}\left(\mathbb{R}^{n}\right)} \leq \frac{\epsilon}{2 N_{0}}
$$

where $N_{0}>0$ is defined as in the proof of (i). Now, by Theorem 2.3, we see that

$$
t^{\frac{\sigma}{2 \alpha}}\left\|e^{-(-\Delta)^{s} t}\left(u_{0}-g\right)\right\|_{L_{q_{1}, \cdots, q_{n}}\left(\mathbb{R}^{n}\right)} \leq N\left\|u_{0}-g\right\|_{L_{p_{1}, \cdots, p_{n}}\left(\mathbb{R}^{n}\right)} \leq \frac{\epsilon}{2} .
$$


On the other hand, using the first assertion in (i) again, we also obtain

$$
t^{\frac{\sigma}{2 \alpha}}\left\|e^{-(-\Delta)^{s} t} g\right\|_{L_{q_{1}, \cdots, q_{n}}\left(\mathbb{R}^{n}\right)} \leq N t^{\frac{\sigma}{2 \alpha}}\|g\|_{L_{q_{1}, \cdots, q_{n}}\left(\mathbb{R}^{n}\right)} \rightarrow 0 \quad \text { as } t \rightarrow 0^{+} .
$$

Then, combine the last two estimates, we infer that there is small number $\delta_{0}=\delta_{0}(\epsilon)>0$ such that

$$
t^{\frac{\sigma}{2 \alpha}}\left\|e^{-(-\Delta)^{s} t} u_{0}\right\|_{L_{q_{1}, \cdots, q_{n}}\left(\mathbb{R}^{n}\right)} \leq \epsilon, \quad \forall t \in\left(0, \delta_{0}\right)
$$

This implies that

$$
\lim _{t \rightarrow 0^{+}} t^{\frac{\sigma}{2 \alpha}}\left\|e^{-(-\Delta)^{s}} u_{0}\right\|_{L_{q_{1}, \cdots, q_{n}}\left(\mathbb{R}^{n}\right)}=0
$$

as desired. The proof is completed.

\section{Proof of Theorem 1.1}

By Duhamel's principle, Eq. (1.1) is recast in the following abstract form

$$
u(t)=u_{0}(t)+\mathcal{B}(u, u)(t),
$$

where

$$
u_{0}(t)=e^{-(-\Delta)^{\alpha} t} \theta_{0}, \quad \mathcal{B}(u, v)(t)=\int_{0}^{t} e^{-(-\Delta)^{\alpha}(t-s)} \mathcal{R}(u(s)) \cdot \nabla v(s) d s .
$$

Our goal is to prove the existence and uniqueness of a fixed point for Eq. (3.1). To proceed, we need to control the nonlinear term. Our next lemma gives some important estimates in mixed norm for the bilinear term $\mathcal{B}(u, v)$ defined in (3.2).

Lemma 3.1. Let $p_{k} \in(1, \infty)$ and $\tau_{k}, \beta_{k}, \gamma_{k} \in(0,1]$ be given numbers satisfying

$$
\gamma_{k} \leq \tau_{k}+\beta_{k} \leq p_{k}, \quad k=1,2 \text {. }
$$

Let

$$
\tau=\sum_{k=1}^{2} \frac{\tau_{k}}{p_{k}}, \quad \beta=\sum_{k=1}^{2} \frac{\beta_{k}}{p_{k}}, \quad \text { and } \quad \gamma=\sum_{k=1}^{2} \frac{\gamma_{k}}{p_{k}} .
$$

Then

$$
\begin{aligned}
& \|\mathcal{B}(u, v)(t)\|_{L_{\frac{p_{1}}{\gamma_{1}}, \frac{p_{2}}{\gamma_{2}}}\left(\mathbb{R}^{2}\right)} \leq N \int_{0}^{t}(t-s)^{-\frac{\tau+\beta-\gamma}{2 \alpha}}\|u(s)\|_{\frac{p_{\frac{1}{\tau_{1}}}, \frac{p_{2}}{\tau_{2}}}{2}\left(\mathbb{R}^{2}\right)}\|\nabla v(s)\|_{L_{\frac{p_{1}}{\beta_{1}}, \frac{p_{2}}{\beta_{2}}}\left(\mathbb{R}^{2}\right)} d s, \\
& \|\nabla \mathcal{B}(u, v)(t)\|_{L_{\frac{p_{1}}{\gamma_{1}}, \frac{p_{2}}{\gamma_{2}}}\left(\mathbb{R}^{2}\right)} \leq N \int_{0}^{t}(t-s)^{-\frac{1+\tau+\beta-\gamma}{2 \alpha}}\|u(s)\|_{L_{\frac{p_{1}}{\tau_{1}}, \frac{p_{2}}{2}}\left(\mathbb{R}^{2}\right)}\|\nabla v(s)\|_{L_{\frac{p_{1}}{\beta_{1}}, p_{2}}\left(\mathbb{R}_{2}\right)} d s,
\end{aligned}
$$

where $N$ is a positive number depending only on $n, \alpha, p_{k}, \alpha_{l}, \beta_{k}, \alpha_{k}$ for $k=1,2$. 
Proof. We only prove the first assertion in the lemma as the proof of the second one can be done similarly. By applying (2.6) of Theorem 2.3, we see that

$$
\|\mathcal{B}(u, v)(t)\|_{\left.\frac{p_{1}, \frac{p_{2}}{\gamma_{1}}, \frac{\mathbb{\gamma}_{2}}{\gamma_{2}}}{2}\right)} \leq N \int_{0}^{t}(t-s)^{-\frac{\tau+\beta-\gamma}{2 \alpha}}\|\mathcal{R}(u(s)) \cdot \nabla v(s)\|_{L_{\frac{p_{1}}{\tau_{1}+\beta_{1}}, \frac{p_{2}}{\tau_{2}+\beta_{2}}}\left(\mathbb{R}^{2}\right)} d s .
$$

Then, as

$$
\frac{\tau_{k}+\beta_{k}}{p_{k}}=\frac{\tau_{k}}{p_{k}}+\frac{\beta_{k}}{p_{k}} \quad \text { for all } k=1,2,
$$

we can repeatedly apply the Hölder's inequality for each integration with respect to each variable $x_{k}$ to find that

$$
\|\mathcal{R}(u(s)) \cdot \nabla v(s)\|_{L \frac{p_{1}}{\tau_{1}+\beta_{1}}, \frac{p_{2}}{\tau_{2}+\beta_{2}}}\left(\mathbb{R}^{2}\right) \leq\|\mathcal{R}(u(s))\|_{L \frac{p_{1}}{\tau_{1}}, \frac{p_{2}}{\tau_{2}}}\left(\mathbb{R}^{2}\right)\|\nabla v(s)\|_{L \frac{p_{1}}{\beta_{1}}, \frac{p_{2}}{\beta_{2}}}\left(\mathbb{R}^{2}\right) .
$$

Therefore

$$
\|\mathcal{B}(u, v)(t)\|_{L_{\frac{p_{1}}{\gamma_{1}}, \frac{p_{2}}{\gamma_{2}}}\left(\mathbb{R}^{2}\right)} \leq N \int_{0}^{t}(t-s)^{-\frac{\tau+\beta-\gamma}{2 \alpha}}\|\mathcal{R}(u(s))\|_{\left.\frac{p_{\frac{1}{1}}, \frac{p_{2}}{\tau_{1}}\left(\mathbb{R}^{2}\right)}{2 \alpha}\|\nabla v(s)\|_{L \frac{p_{1}}{\beta_{1}, \frac{p_{2}}{\beta_{2}}}} \| \mathbb{R}^{2}\right)} d s .
$$

The first assertion of the lemma follows from this last estimate and the boundedness of the Riesz transform in Theorem 2.1. The proof is completed.

Now, to prove Theorem 1.1, let us recall the following useful abstract lemma whose proof is based on the Picard's interation.

Lemma 3.2. Let $X$ be a Banach space with norm $\|\cdot\|_{X}$. Let $\mathcal{B}: X \times X \rightarrow X$ be a bilinear map such that there is $N_{0}>0$ so that

$$
\|\mathcal{B}(u, v)\|_{X} \leq N_{0}\|u\|_{X}\|v\|_{X}, \quad \forall u, v \in X
$$

Then, for every $u_{0} \in X$ with $4 N_{0}\left\|u_{0}\right\|_{X}<1$, the equation

$$
u=u_{0}+\mathcal{B}(u, u)
$$

has unique solution $u \in X$ with

$$
\|u\|_{X} \leq 2\left\|u_{0}\right\|_{X}
$$

We are now ready to provide the proof of Theorem 1.1.

Proof of Theorem 1.1. Let $p=\left(p_{1}, p_{2}\right), q=\left(q_{1}, q_{2}\right)$ be as in the Theorem 1.1. Let $\theta_{0} \in$ $L_{p_{1}, p_{2}}\left(\mathbb{R}^{2}\right)$ and recall that

$$
\delta=\frac{1}{q_{1}}+\frac{1}{q_{2}} \in(0,2 \alpha-1) .
$$


We now prove (i). Recall the definitions of $x_{p, q, \infty}$ and $y_{p, q, \infty}$ in (1.5) and (1.6). We apply Lemma 3.2 to obtain the existence and uniqueness of solution of (3.1) in $x_{p, q, \infty}$. Then, we prove that the solution $u$ belongs to $y_{p, q, \infty}$ To this end, we begin with the proof that $u_{0} \in X_{p, q, \infty}$. From Theorem 2.3 and the definition of $u_{0}$ in (3.2), we have

$$
\begin{aligned}
& \left\|u_{0}(t)\right\|_{L_{q_{1}, q_{2}}\left(\mathbb{R}^{2}\right)} \leq N_{1} t^{-\frac{(2 \alpha-1)-\delta}{2 \alpha}}\left\|\theta_{0}\right\|_{L_{p_{1}, p_{2}}\left(\mathbb{R}^{2}\right),}, \\
& \left\|\nabla u_{0}(t)\right\|_{L_{p_{1}, p_{2}}\left(\mathbb{R}^{2}\right)} \leq N_{1} t^{-\frac{1}{2 \alpha}}\left\|\theta_{0}\right\|_{L_{p_{1}, p_{2}}\left(\mathbb{R}^{2}\right)}, \quad \forall t>0,
\end{aligned}
$$

where $N_{1}>0$ is a universal constant depending only on $n, p$ and $q$. Moreover, it follows from Lemma 2.1 that $t^{\frac{(2 \alpha-1)-\delta}{2 \alpha}} e^{-(-\Delta)^{\alpha} t}$. is uniformly bounded from $L_{p_{1}, p_{2}}\left(\mathbb{R}^{2}\right)$ to $L_{q_{1}, q_{2}}\left(\mathbb{R}^{2}\right)$ and tends to zero as $t \rightarrow 0^{+}$, we see that $\frac{\frac{(2 \alpha-1)-\delta}{2}}{2} u_{0}$ vanishes as $t=0$. Similarly, as $t^{\frac{1}{2 \alpha}} \nabla e^{-(\Delta)^{\alpha} t}$. is uniformly bounded from $L_{p_{1}, p_{2}}\left(\mathbb{R}^{2}\right)$ to $L_{p_{1}, p_{2}}\left(\mathbb{R}^{2}\right)^{2}$ and tends to zero as $t \rightarrow 0^{+}$, we also have $t^{\frac{1}{2 \alpha}} \nabla u_{0}$ equals to zero as $t \rightarrow 0^{+}$. In conclusion, we have shown that $u_{0} \in X_{p, q, \infty}$ and

$$
\left\|u_{0}\right\| x_{p, q, \infty} \leq N_{1}\left\|\theta_{0}\right\|_{L_{p_{1}, p_{2}}\left(\mathbb{R}^{2}\right)}
$$

It now remains to prove that the bilinear form $\mathcal{B}: x_{p, q, \infty} \times x_{p, q, \infty} \rightarrow x_{p, q, \infty}$ is bounded. By (1.3), (1.7), and (3.3), we are able to apply the first assertion in Lemma 3.1 with $\beta_{k}=1$ and $\gamma_{k}=\tau_{k}=\frac{p_{k}}{q_{k}} \in(0,1]$ to find that

$$
\begin{aligned}
& \|\mathcal{B}(u, v)(t)\|_{L_{q_{1}, q_{2}}\left(\mathbb{R}^{2}\right)} \\
\leq & N \int_{0}^{t}(t-s)^{-\frac{2 \alpha-1}{2 \alpha}}\|u(s)\|_{L_{q_{1}, q_{2}}\left(\mathbb{R}^{2}\right)}\|\nabla v(s)\|_{L_{p_{1}, p_{2}}\left(\mathbb{R}^{2}\right)} d s \\
\leq & N\|u\|_{x_{p, q, \infty}}\|v\|_{x_{p, q, \infty}} \int_{0}^{t}(t-s)^{-\frac{2 \alpha-1}{2 \alpha}} s^{-\frac{2 \alpha-\delta}{2 \alpha}} d s .
\end{aligned}
$$

To control the integration in the last estimate, we split it into two time intervals $(0, t / 2)$ and $(t / 2, t)$. We then obtain

$$
\begin{aligned}
& \|\mathcal{B}(u, v)(t)\|_{L_{q_{1}, q_{2}}\left(\mathbb{R}^{2}\right)} \\
\leq & N\|u\|_{x_{p, q, \infty}}\|v\|_{x_{p, q, \infty}}\left[\int_{0}^{t / 2}(t-s)^{-\frac{2 \alpha-1}{2 \alpha}} s^{-\frac{2 \alpha-\delta}{2}} d s+\int_{t / 2}^{t}(t-s)^{-\frac{2 \alpha-1}{2 \alpha}} s^{-\frac{2 \alpha-\delta}{2}} d s\right] \\
\leq & N\|u\|_{x_{p, q, \infty}}\|v\|_{x_{p, q, \infty}}\left[t^{-\frac{2 \alpha-1}{2 \alpha}} \int_{0}^{t / 2} s^{-\frac{2 \alpha-\delta}{2 \alpha}} d s+t^{-\frac{2 \alpha-\delta}{2 \alpha}} \int_{t / 2}^{t}(t-s)^{-\frac{2 \alpha-1}{2 \alpha}} d s\right] \\
\leq & N t^{-\frac{(2 \alpha-1)-\delta}{2 \alpha}}\|u\|_{x_{p, q, \infty}}\|v\|_{x_{p, q, \infty} .}
\end{aligned}
$$

Similarly, from (1.3), (1.7), (3.3), we can apply the second assertion in Lemma 3.1 with 


$$
\begin{aligned}
\gamma_{k}=1, & \beta_{k}=1 \text { and } \tau_{k}=\frac{p_{k}}{q_{k}} \in(0,1] \text { to obtain } \\
& \|\nabla \mathcal{B}(u, v)(t)\|_{L_{p_{1}, p_{2}}\left(\mathbb{R}^{2}\right)} \\
\leq & N \int_{0}^{t}(t-s)^{-\frac{1+\delta}{2 \alpha}}\|u(s)\|_{L_{q_{1}, q_{2}}\left(\mathbb{R}^{2}\right)}\|\nabla u(s)\|_{L_{p_{1}, p_{2}}\left(\mathbb{R}^{2}\right)} d s \\
\leq & N\|u\|_{x_{p, q, \infty}}\|v\| x_{p_{p, q, \infty}} \int_{0}^{t}(t-s)^{-\frac{1+\delta}{2 \alpha}} s^{-1+\frac{\delta}{2 \alpha}} d s \\
= & N\|u\|_{x_{p, q, \infty}}\|v\|_{x_{p, q, \infty}}\left[\int_{0}^{t / 2}(t-s)^{-\frac{1+\delta}{2 \alpha}} s^{-1+\frac{\delta}{2 \alpha}} d s+\int_{t / 2}^{t}(t-s)^{-\frac{1+\delta}{2 \alpha}} s^{-1+\frac{\delta}{2 \alpha}} d s\right] \\
= & N\|u\|_{x_{p, q, \infty}}\|v\| x_{x_{p, q, \infty}}\left[t^{-\frac{1+\delta}{2 \alpha}} \int_{0}^{t / 2} s^{-1+\frac{\delta}{2 \alpha}} d s+t^{-1+\frac{\delta}{2 \alpha}} \int_{t / 2}^{t}(t-s)^{-\frac{1+\delta}{2 \alpha}} d s\right] \\
\leq & N t^{-\frac{1}{2 \alpha}}\|u\|_{x_{p, q, \infty}}\|v\|_{x_{p, q, \infty}} .
\end{aligned}
$$

From the last two estimates and the definition of $\mathcal{B}(u, v)$ and Lemma 2.1, it follows that $t^{\frac{(2 \alpha-1)-\delta}{2 \alpha}} \mathcal{B}(u, v):[0, \infty) \rightarrow L_{q_{1}, q_{2}}\left(\mathbb{R}^{2}\right)$ is continuous and vanishes at $t=0$. Similarly, we can also prove that $t^{\frac{1}{2 \alpha}} \nabla \mathcal{B}(u, v):[0, \infty) \rightarrow L_{p_{1}, p_{2}}\left(\mathbb{R}^{2}\right)$ is continuous and vanishes as $t \rightarrow 0^{+}$. Therefore, we conclude that $\mathcal{B}(u, v) \in X_{p, q, \infty}$ and

$$
\|\mathcal{B}(u, v)\|_{x_{p, q, \infty}} \leq N_{2}\|u\|_{x_{p, q, \infty}}\|v\|_{x_{p, q, \infty},} \quad \forall u, v \in X_{p, q, \infty},
$$

where $N_{2}$ is a constant depending only on $n, p$ and $q$. In other words, the bilinear form $\mathcal{B}: x_{p, q, \infty} \times x_{p, q, \infty} \rightarrow x_{p, q, \infty}$ is bounded.

Next, let us choose $\lambda_{0}>0$ and sufficiently small so that

$$
4 N_{1} N_{2} \lambda_{0}<1,
$$

where $N_{1}$ is defined in (3.4), and $N_{2}$ is defined in (3.6). Note that both of these numbers depend only on $p, q$ and $n$. Now, if $\left\|\theta_{0}\right\|_{L_{p_{1}, p_{2}}\left(\mathbb{R}^{2}\right)} \leq \lambda_{0}$, then it follows from (3.4) that

$$
4 N_{2}\left\|u_{0}\right\| x_{p, q, \infty} \leq 4 N_{1} N_{2}\left\|a_{0}\right\|_{L_{p_{1}, \cdots, p_{n}}\left(\mathbb{R}^{n}\right)} \leq 4 N_{1} N_{2} \lambda_{0}<1 .
$$

From this and by applying Lemma 3.2, we can find a unique solution $u \in x_{p, q, \infty}$ of Eq. (3.1) such that

$$
\|u\|_{x_{p, q, \infty}} \leq 2\left\|u_{0}\right\|_{X_{\infty}} \leq 2 N_{1}\left\|\theta_{0}\right\|_{L_{p_{1}, p_{2}}\left(\mathbb{R}^{2}\right)} .
$$

Now, to complete the proof (i), we need to show that $u \in y_{p, \infty}$. We recall that the definition of $y_{p, \infty}$ is given in (1.6). Since

$$
u(t)=u_{0}(t)+\mathcal{B}(u, u)(t),
$$

we have

$$
\begin{aligned}
& \|u(t)\|_{L_{p_{1}, p_{2}}\left(\mathbb{R}^{2}\right)} \leq\left\|u_{0}(t)\right\|_{L_{p_{1}, p_{2}}\left(\mathbb{R}^{2}\right)}+\|\mathcal{B}(u, u)(t)\|_{L_{p_{1}, p_{2}}\left(\mathbb{R}^{2}\right)} \\
& \|\nabla u(t)\|_{L_{p_{1}, p_{2}}\left(\mathbb{R}^{2}\right)} \leq\left\|\nabla u_{0}(t)\right\|_{L_{p_{1}, p_{2}}\left(\mathbb{R}^{2}\right)}+\|\nabla \mathcal{B}(u, u)(t)\|_{L_{p_{1}, p_{2}}\left(\mathbb{R}^{2}\right)}
\end{aligned}
$$


Then, by applying Lemma 2.3, we see that

$$
\begin{aligned}
& \left\|u_{0}(t)\right\|_{L_{p_{1}, p_{2}}\left(\mathbb{R}^{2}\right)} \leq N\left\|\theta_{0}\right\|_{L_{p_{1}, p_{2}}\left(\mathbb{R}^{2}\right)}, \\
& \left\|\nabla u_{0}(t)\right\|_{L_{p_{1}, p_{2}}\left(\mathbb{R}^{2}\right)} \leq N t^{-\frac{1}{2 \alpha}}\left\|\theta_{0}\right\|_{L_{p_{1}, p_{2}}\left(\mathbb{R}^{2}\right)} .
\end{aligned}
$$

On the other hand, by (1.3), (1.7), and (3.3), we are able to apply the first assertion in Lemma 3.1 with $\gamma_{k}=1, \tau_{k}=\frac{p_{k}}{q_{k}} \in(0,1]$ and $\beta_{k}=1$ to infer that

$$
\begin{aligned}
& \|\mathcal{B}(u, u)(t)\|_{L_{p_{1}, p_{2}}\left(\mathbb{R}^{2}\right)} \\
\leq & N \int_{0}^{t}(t-s)^{-\frac{\delta}{2 \alpha}}\|u(s)\|_{L_{q_{1}, q_{2}}\left(\mathbb{R}^{2}\right)}\|\nabla u(s)\|_{L_{p_{1}, p_{2}}\left(\mathbb{R}^{2}\right)} d s \\
\leq & N\|u\|_{x_{p, q, \infty}}^{2} \int_{0}^{t}(t-s)^{-\frac{\delta}{2 \alpha}} s^{-\left(1-\frac{\delta}{2 \alpha}\right)} d s \\
= & N\|u\|_{x_{p, q, \infty}}^{2}\left[\int_{0}^{t / 2}(t-s)^{-\frac{\delta}{2 \alpha}} s^{-\left(1-\frac{\delta}{2 \alpha}\right)} d s+\int_{t / 2}^{t}(t-s)^{-\frac{\delta}{2 \alpha}} S^{-\left(1-\frac{\delta}{2 \alpha}\right)} d s\right] \\
= & N\|u\|_{x_{p, q, \infty}}^{2}\left[t^{-\frac{\delta}{2 \alpha}} \int_{0}^{t / 2} s^{-\left(1-\frac{\delta}{2 \alpha}\right)} d s+t^{-\left(1-\frac{\delta}{2 \alpha}\right)} \int_{t / 2}^{t}(t-s)^{-\frac{\delta}{2 \alpha}} d s\right] \\
\leq & N\left\|\theta_{0}\right\|_{L_{p_{1}, p_{2}}\left(\mathbb{R}^{2}\right)^{\prime}}^{2}
\end{aligned}
$$

where in the last estimate, we used (3.8). Also, by (3.5) and (3.8), it follows that

$$
\|\nabla \mathcal{B}(u, u)\|_{L_{p_{1}, p_{2}}\left(\mathbb{R}^{2}\right)} \leq N t^{-\frac{1}{2 \alpha}}\|u\|_{\hat{X}_{p, q, \infty}}^{2} \leq N t^{-\frac{1}{2 \alpha}}\left\|\theta_{0}\right\|_{L_{p_{1}, p_{2}}\left(\mathbb{R}^{2}\right)}^{2}
$$

Then, from the estimates (3.9), (3.10), (3.11), (3.12) and the fact that $\left\|\theta_{0}\right\|_{L_{p_{1}, p_{2}}\left(\mathbb{R}^{2}\right)}$ is sufficiently small that, we see that

$$
\|u\|_{y_{p, \infty}} \leq N_{0}\left\|\theta_{0}\right\|_{L_{p_{1}, p_{2}}\left(\mathbb{R}^{2}\right)}
$$

The proof of (i) is therefore completed.

Now, we turn to prove (ii). As in the proof of (3.4), we see that $u_{0} \in X_{p, q, \infty}$. From the definition of the norm of the space $X_{p, q, \infty}$ in (1.5), the continuity and the vanishes of $t^{\frac{1-\delta}{2 \alpha}} u_{0}$ and of $t^{\frac{1}{2 \alpha}} \nabla u_{0}$ at $t=0$, we can choose a sufficiently small number $T_{0}>0$ depending on $n, p, q$ and $\theta_{0}$ so that

$$
\left\|u_{0}\right\|_{x_{p, q, T_{0}}} \leq \lambda_{0}
$$

where $\lambda_{0}$ is defined as in (3.7). Moreover, by following the proof of (3.6), we can also see that the bilinear form $\mathcal{B}: x_{p, q, T_{0}} \times x_{p, q, T_{0}} \rightarrow x_{p, q, T_{0}}$ is bounded with

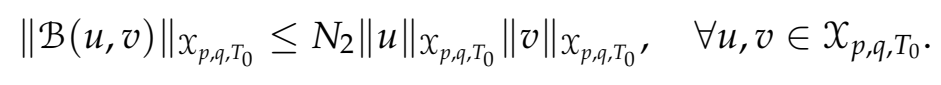


Then, applying Lemma 3.2 again, we can find a unique local time solution $u \in X_{p, q, T_{0}}$ of (3.1) satisfying

$$
\|u\|_{x_{p, q, T_{0}}} \leq 2 N_{1}\left\|\theta_{0}\right\|_{L_{p_{1}, p_{2}}\left(\mathbb{R}^{2}\right)} .
$$

Now, we only need to prove that the solution $u$ that we found is indeed in $y_{p, T_{0}}$. However, this can be done exactly as in the proof that $u \in y_{p, \infty}$ in (i), and we skip it. The proof of the theorem is then completed.

\section{Acknowledgements}

T. Phan's research is partially supported by the Simons Foundation, grant \# 354889. The authors would like to thank the anonymous referees for valuable comments.

\section{References}

[1] A. Benedek and R. Panzone, The space $L^{p}$, with mixed norm, Duke Math. J., 28(3) (1961), 301-324.

[2] R. M. Blumenthal, and R. K. Getoor, Some theorems on stable processes, Trans. Amer. Math. Soc., 95 (1960), 263-273.

[3] K. Bogdan, and T. Jakubowski, Estimates of the heat kernel of fractional Laplacian perturbed by gradient operators, Commun. Math. Phys., 271(1) (2007), 179-198.

[4] L. Brandolese, and G. Karch, Far field asymptotics of solutions to convection equation with anomalous diffusion, J. Evol. Equations, 8(2) (2008), 307-326.

[5] L. A. Caffarelli, and A. Vasseur, Drift diffusion equations with fractional diffusion and the quasi-geostrophic equation, Ann. Math., 171(3) (2010), 1903-1930.

[6] J. Carrillo, and L. Ferreira, The asymptotic behaviour of subcritical dissipative quasigeostrophic equations, Nonlinearity, 21(5) (2008), 1001-1018.

[7] P. Constantin, A. J. Majda, and E. G. Tabak, Singular front formation in a model for quasigeostrophic flow, Phys. Fluids, 6(1) (1994), 9-11.

[8] P. Constantin, A. J. Majda, and E. G. Tabak, Formation of strong fronts in the 2-D quasigeostrophic thermal active scalar, Nonlinearity, 7(6) (1994), 1495-1533.

[9] P. Constantin, G. Iyer, and J. Wu, Global regularity for a modified critical dissipative quasigeostrophic equation, Indiana Univ. Math. J., 57(6) (2008), 2681-2692.

[10] P. Constantin, and J. $\mathrm{Wu}$, Behavior of solutions of 2D quasi-geostrophic equations, SIAM J. Math. Anal., 30(5) (1999), 937-948.

[11] P. Constantin, and J. Wu, Hölder continuity of solutions of supercritical dissipative hydrodynamic transport equations, Ann. Inst. H. Poincare Anal. Non Linaire, 26(1) (2009), 159-180.

[12] P. Constantin, and J. Wu, Regularity of Hölder continuous solutions of the supercritical quasi-geostrophic equation, Ann. Inst. H. Poincare Anal. Non Linaire, 25(6) (2008), 11031110.

[13] A. Córdoba, and D. Córdoba, A maximum principle applied to quasi-geostrophic equations, Commun. Math. Phys., 249(3) (2004), 511-528.

[14] D. V. Cruz-Uribe, J. M. Martell, and C. Pérez. Weights, Extrapolation and the Theory of Rubio de Francia, volume 215 of Operator Theory: Advances and Applications. Birkhäuser/Springer Basel AG, Basel, 2011. 
[15] H. Dong, and D. Kim, On $L_{p}$-estimates for elliptic and parabolic equations with $A_{p}$ weights, Trans. Amer. Math. Soc., 370(7) (2018), 5081-5130.

[16] H. Dong, and D. Li, Spatial analyticity of the solutions to the subcritical dissipative quasigeostrophic equations, Arch. Ration. Mech. Anal., 189(1) (2008), 131-158.

[17] J. García-Cuerva, and J. L. Rubio de Francia, Weighted norm inequalities and related topics, North-Holland Mathematics Studies, 116, Notas de Matemática, 104, North-Holland Publishing Co., Amsterdam, 1985.

[18] A. Kiselev, Small scales and singularity formation in fluid dynamics, proceedings ICM 2018, arXiv:1807.00184.

[19] A. Kiselev, F. Nazarov, and A. Volberg, Global well-posedness for the critical 2D dissipative quasi-geostrophic equation, Invent. Math., 167(3) (2007), 445-453.

[20] V. N. Kolokoltsov, Symmetric stable laws and stable-like jump-diffusions, Lond. Math. Soc., 80 (2000), 725-768.

[21] N. V. Krylov, Rubio de Francia extrapolation theorem and related topics in the theory of elliptic and parabolic equations: A survey, St. Petersburg Math. J., to appear, arXiv:1901.00549.

[22] H. Miura, Dissipative quasi-geostrophic equation for large initial data in the critical Sobolev space, Commun. Math. Phys., 267(1) (2006), 141-157.

[23] T. Phan, Well-posedness for the Navier-Stokes equations in critical mixed-norm Lebesgue spaces, J. Evolution Equations, DOI: 10.1007/s00028-019-00529-1.

[24] L. Silvestre, Eventual regularization for the slightly supercritical quasi-geostrophic equation, Ann. Inst. H. Poincare Anal. Non Linaire, 27(2) (2010), 693-704.

[25] E. M. Stein, Singular Integrals and Differentiability Properties of Functions, Princeton Mathematical Series, No. 30, Princeton University Press, Princeton, N.J. 1970. 